\title{
NASA-TM-88239
}

NASA Technical Memorandum 88239

\section{Liquid Helium Pumps for In-Orbit Transfer}

\section{Peter Kittel}

April 1986

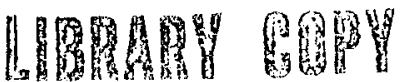

MAY 13986

LANGLEY RESEARCH CENTER

I.IBRARY, NASA

HAMPTCN, VIRGINIA

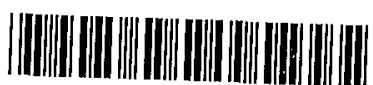

NF00948

\section{N/SA}

National Aeronautics and

Space Administration 
NASA Technical Memorandum 88239

\section{Liquid Helium Pumps for In-Orbit Transfer}

Peter Kittel, Ames Research Center, Moffett Field, California

April 1986

\section{N/SA}

National Aeronautics and

Space Administration

Ames Research Center

Moffett Field, California 94035

N86-31890 tt 


\section{LIQUID HELIUM PUMPS FOR IN-ORBIT TRANSFER}

Peter Kittel

NASA Ames Research Center, Moffett Field, CA 94035, USA

Abstract

Both mechanical and fountain-effect pumps are being considered for use in the in-orbit resupply of superfluid helium to a number of scientific instrument systems. This paper presents a review of the operating characteristics of these pumps. Particular emphasis will be given to the different methods of evaluating the efficiency of these pumps and their effectiveness in a transfer system.

Nomenclature

$\begin{array}{ll}\text { C } & \text { heat capacity } \\ \text { H } & \text { enthalpy } \\ \text { L } & \text { latent heat } \\ \text { P } & \text { pressure } \\ \text { Q } & \text { heat flux } \\ \text { S } & \text { entropy } \\ \text { T } & \text { temperature } \\ \text { V } & \text { volume } \\ \text { W } & \text { fluid power } \\ \text { m } & \text { mass } \\ \dot{m} & \text { mass flow } \\ \mathrm{n} & \text { number of stages } \\ \mathrm{V} & \text { velocity } \\ \varepsilon & \text { efficiency }\end{array}$




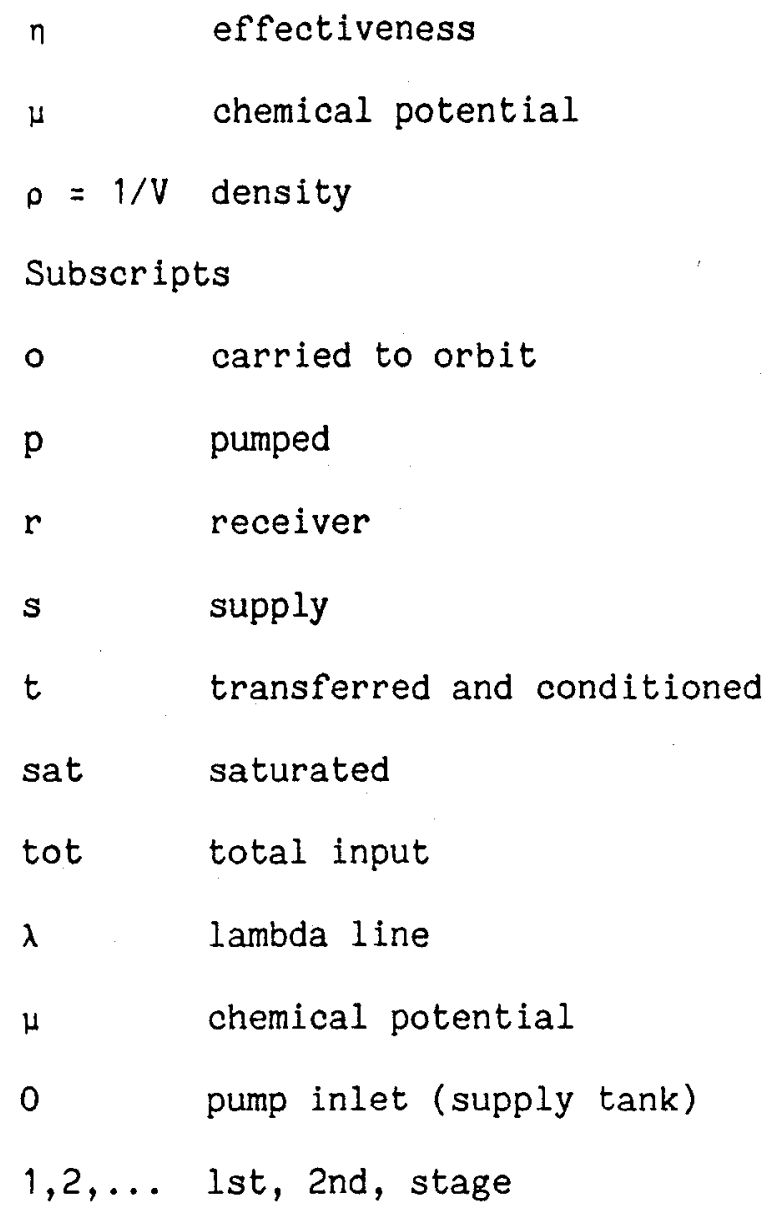

The ability to resupply orbiting satellites with superfluid helium would extend the useful life of several instrument systems currently being developed. These include the Space Infrared Telescope Facility, the Advanced X-ray Astrophysics Facility, Gravity Probe-B, the Superconducting Magnet Facility, and the Large Deployable Reflector. Several methods of replenishing orbiting satellites with superfluid helium have been considered. ${ }^{1-4}$ The most promising approach, which is being developed, is a pumped transfer using either a mechanical pump (MP) or a fountain-effect (thermomechanical effect) pump (FEP). This paper describes these pumps and discusses their efficiencies. Several measures of efficiencies and effectiveness have been used by various 
authors. 1-9 The differences will be discussed here. Finally, a method of increasing the pressure head of an FEP by using a multistage pump will be discussed.

The basic components of a transfer system are a Dewar supply tank, a transfer line, a Dewar receiving tank, and a pump. The pump is mounted inside the supply tank. (Descriptions of possible resupply systems $1,3,4,10$ and of proposed demonstrations on the Space Shuttle 2,11 are given in the literature. The pump will be required to operate in several modes: 1) the cooldown of the transfer line prior to transfer; 2) the filling of an already cold or partially full tank; and 3) the cool-down and subsequent filling of a warm tank. The latter two modes represent the extremes of operation. The filling of a cold tank will require flow rates of up to $0.5 \mathrm{~m}^{3} / \mathrm{h}(20 \mathrm{~g} / \mathrm{s})$ with a pressure head of about $500 \mathrm{~Pa}$. The cooling of a warm tank results in a lower flow rate, but the system back pressure will be higher. At present, we estimate a flow rate of $\approx 0.05 \mathrm{~m}^{3} / \mathrm{h}(2 \mathrm{~g} / \mathrm{s})$ at $30 \mathrm{kPa}$ would be necessary to start cooling a $150 \mathrm{~K}$ tank. Both the MP and the FEP should be able to meet these requirements. In the following discussion, the emphasis will be on the second mode--filling a cold tank.

Mechanical pump

The mechanical pump being considered for this application is a centrifugal pump driven by a three-phase induction motor. One of the uncertainties involved in using a centrifugal pump in HeII is the effect of the superfluid properties on the pump performance. It has been postulated that the lack of viscosity in the superfluid component would prevent HeII being pumped. To study this postulate, a centrifugal pump (Fig. 1) (which was developed for other purposes) has been tested in liquid helium. Early tests of this pump 5,6 
in HeI and HeII have shown that HeII can be pumped. Although the actual data were limited, these early tests did show that the pump performance was consistent with the standard scaling laws for pump performance. This result probably occurred because the velocities within the pump were several orders of magnitude greater than the critical velocity. Hence, mutual friction causes the superfluid component of the He to be entrained by the normal fluid. The entrainment does not suppress the superfluid properties, but causes the difference of the average velocities of the two components to be small. The performance data in HeI have been scaled 8 (Fig. 2) to predict the performance with HeII. The peak efficiency (including pump and motor losses) should be $33 \%$ with a $20-\mathrm{kPa}$ head and a $0.8-\mathrm{m}^{3} / \mathrm{h}$ flow. This prediction is currently being tested. It should be noted that the impeller of this pump has not been optimized for HeII nor for the pressure and flow requirements of this application. An optimized pump will be built after the scaling laws have been verified. The efficiency of an optimized pump may be a factor of two higher than that of the present pump. 12

Fountain-effect pump

The superfluid properties of HeII are essential for the operation of fountain effect pumps. The behavior of the pumps is well understood whenever the internal velocities are near or below the critical velocity. ${ }^{16,17}$ An FEP is a simple device (Fig. 3). It consists of a block (plug) of porous material and a heater. A stainless steel porous plug $2.5 \mathrm{~cm}$ thick and $7 \mathrm{~cm}$ in diameter with a porosity of 0.4 and pores of $1.5 \mu \mathrm{m}$ is thought to be adequate for resupplying liquid helium. ${ }^{2}$ Such high flow rates have not yet been demonstrated. Heat that is applied to the downstream side causes the superfluid component to flow through the porous plug while the normal fluid component is 
immobilized by viscosity. If an ideal "superleak" is used as a pump, its behavior is governed by

$$
\mathrm{dP} / \mathrm{dT}=\rho \mathrm{S}
$$

and

$$
Q_{1}=\dot{m} S\left(T_{1}\right) T_{1}
$$

These are the thermomechanical and mechanocaloric effects, respectively. Equation (1) states that the process occurs at a constant chemical potential. Equation (2) shows the result of the superfluid component carrying no entropy. In an actual pump, the terms representing the thermoconductivity (normal fluid leakage) through the plug and forced convection of heat away from the heater must be added to the right side of (2). Above the critical velocity, these equations must be further modified 15 to account for mutual friction. In the proposed application, the pump will be selected to keep the velocities within the pump subcritical and to keep the other losses small so that (1) and (2) provide a good description of the pump's performance (as shown in Fig. 4). The deviation at sizable temperature differences from the constant chemical-potential curve is the result of mutual friction. As the pump depends on the superfluid properties, the temperatures must be kept below the lambda line. Thus the largest pressure that can be generated is indicated by the point on the lambda line where

$$
\mu\left(P_{\lambda}, T_{\lambda}\right)=\mu\left(P_{0}, T_{0}\right)
$$

In practice, pressures of up to $\approx 50 \mathrm{kPa}$ have been observed. 


\section{Efficiency}

Several different definitions of efficiency have been used to evaluate the performance of helium transfer systems. These include a hydrothermodynamic efficiency of the pump, 1,5,6 a mass-transfer efficiency, ${ }^{1,2}$ and an efficiency derived from treating the process as a thermodynamic cycle. 7,9 These three efficiencies will be compared in this section.

The hydrothermodynamic efficiency, which is the normal efficiency of a pump, was the first one that was used. ${ }^{8}$ This efficiency is defined by

$$
\varepsilon=W / Q_{\text {tot }}
$$

where $W=\dot{m}\left[\Delta P / \rho+\left(v^{2}\right) / 2\right]$ is the fluid power. To evaluate $P$ and $v$, $a$ control volume is first drawn around the pump. $\mathrm{P}$ and $\mathrm{v}$ are then evaluated at the surface of this control volume. Since we intend to have the pump mounted in the supply tank, the most convenient location for the control volume is the region between the liquid-vapor interface in the supply tank and the outlet tube of the pump. In this case, the inlet pressure is equal to the saturated vapor pressure and the inlet velocity is negligible. This is the approach that has been used in MP testing 5,6 and in estimating the performance of such a pump in superfluid helium. ${ }^{8}$ It has also been used to analyze the performance of an FEP. ${ }^{1}$ A comparison of this efficiency for the two types of pumps is shown in Fig. 5. The curves in this figure for the MP are based on the estimated performance of an existing pump. 8 The MP curves include the effects of motor efficiency. ${ }^{6}$ To obtain the curves for the FEP, an ideal superleak is assumed. (For small temperature differences, $\Delta \mathrm{T}$, the efficiency is $\varepsilon \approx \Delta T / T$.) None of the expected internal losses ${ }^{15}$ has been included. Figure 5 shows that the MP is more efficient except at very low flow rates. 
Interestingly, the efficiency of the FEP is not very dependent on the fluid velocity. This is caused partly by omitting the velocity-dependent internal losses which would lower the efficiency.

This type of analysis has been extended ${ }^{7}$ to include contributions caused by thermal conduction along the transfer line back to the pump. Such conduction was found to have only a small effect on the MP, but it could have a dramatic effect on the FEP. For small heat fluxes, the MP is more efficient. However, for high heat fluxes, the FEP is partially or wholly driven by this heat, thereby increasing its efficiency. The efficiency can even exceed 1 ; i.e., the pump is driven by heat conducted back along the transfer line with no additional heat being required. Unfortunately, since such a heat flux is difficult to control, the FEP also becomes difficult to control. We are planning to have such high Reynolds numbers $\left(\sim 10^{6}\right.$ within the transfer line that the heat flux should be small. 18

While the hydrothermodynamic efficiency is a good means to represent pump performance, it is not necessarily the appropriate measure of system performance. The effect on system performance of the temperature rise within the pump is not fully accounted for in the preceding analysis. Ideally, an MP is an isentropic device; while an FEP operates at constant chemical potential. Thus, for ideal pumps acting on HeII, the temperature of the liquid passing through the pump will be decreased by the MP (because $\mathrm{dP} /\left.\mathrm{dT}\right|_{\mathrm{S}}<0$ ) and increased by the FEP (because $\mathrm{dP} /\left.\mathrm{dT}\right|_{\mu}>0$ ). In actual pumps, inefficiencies will cause positive temperature changes for both pumps. The effect on the system performance of the heat dissipated within the pump depends on where the heat goes. Heat that ends up in the supply tank has a different effect than heat that is carried by the fluid to the receiver tank. System performance 
will also be affected by other losses in the system. Thus, the pump inefficiency is just one of several losses.

In the system, another measure of performance is more appropriate. As the purpose of the transfer system is to fill the receiver tank, an appropriate measure is transfer effectiveness:

$$
n=m_{t} / m_{0}
$$

where $m_{t}$ is the mass transferred and conditioned to the desired operating temperature and $m_{0}$ is the mass transported to orbit. Because of conditioning losses (Fig. 6), 1 it is more effective to perform the transfer with HeII rather than transferring $\mathrm{HeI}$ and converting after the transfer. DePirro ${ }^{2}$ was the first to analyze the system losses. He showed that while using an MP may produce a more effective transfer, it is not significantly more effective than an FEP. For either pump, much of the heat is dissipated in the supply tank where some of the liquid is vaporized. For the FEP,-this heat comes from thermal conduction through the pump and from the mechanocaloric effect. For the MP, this heat comes from the motor losses. The pump losses can also contribute if there is a heat exchanger between the pump outlet and the supply tank. Otherwise, it heats the transferred fluid, which must be recooled, causing conditioning losses in the receiver. Some of the heat that is used to drive the FEP is similarly transported to the receiver. By including the various parasitic losses for the pump, tanks, and transfer line, the transfer effectiveness of a system can be determined. This has been done for one of the proposed space demonstrations. 5 The results are shown in Fig. 7 .

The drawbacks of this type of analysis are that it is very systemdependent and is difficult to use when evaluating individual components. For 
individual components, an idealized transfer analys is has been introduced. 9 Here the mass loss (caused by vaporization) is found for a thermodynamic cycle in which the fluid is first pressurized and subcooled by the pump. It is then transferred to the receiver, where it is returned to its original pressure and temperature. This analysis showed that in some situations the FEP will be more effective than the MP. However, the analysis was based on an unproven assumption about the properties of HeII at high flow rates in porous media. It was assumed that the porous material of the pump does not act as an ideal superleak; rather, at flow rates well above the critical velocity, the mutual friction causes "local thermodynamic equilibrium" to be established. This in turn causes the helium to be transported at constant chemical potential. Therefore, the heat needed to drive the fluid through such a supercritical FEP is

$$
Q_{\mu}=\int_{T_{0}^{\prime}}^{T_{1}} \dot{m} C_{\mu} d T
$$

Comparing $Q_{\mu}$ to the heat required to drive the subcritical FEP (2), we see that $Q_{1}>Q_{\mu}$. (Here the terms "subcritical" and "supercritical" refer to the flow state within the FEP.)

The cyclic effectivenesses of various ideal pumps can be compared easily. These pumps are the supercritical FEP, the MP, and the subcritical FEP. The operating cycles of these pumps are shown in Fig. 8. We will analyze the situation in which the pumps all produce the same pressure head. The cycle for the supercritical FEP is composed of the pressurization at constant chemical potential, followed by the isenthalpic flow through the transfer line, and finally the return to the starting conditions by evaporative cooling 
along the saturation curve within the receiver tank. This is shown as path $A-B-C-A$ in Fig. 8. The isenthalpic flow in the transfer line assumes that changes in the kinetic energy of the flow, the internal losses, and the thermal conductivity can all be neglected. 10 The MP follows a similar path, except the pressurization is isentropic. The cycle for the MP is shown as path $A-B^{\prime}-C^{\prime}-A$ in Fig. 8. The subcritical FEP begins with the pressurization by the fountain and mechanocaloric effects. This involves the conversion of normal fluid into superfluid in the supply tank and the reverse conversion on the downstream side of the pump. Once the fluid is pressurized, the process is the same as for the other pumps. This is shown as path $A-A^{\prime}-A^{\prime \prime}-B-C$ in Fig. 8. As the liquid density is nearly constant, each of the pumps will do the same $\int d(P V)$ work. They differ only in the amount of heat, $\int T d S$, and in the amount of mass vaporized. These terms are summarized in Table 1, which lists the mass lost per unit of mass pumped. The transfer effectiveness (5) is given by

$$
\eta=\left(1-\Delta m_{r} / m_{p}\right)\left(1+\Delta m_{s} / m_{p}\right)^{-1}
$$

where $m_{t}=m_{p}-\Delta m_{r}$ and $m_{o}=m_{p}+\Delta m_{s}$. It is clear that mass losses in either tank will reduce the effectiveness. However, because of the exponential nature of $\Delta \mathrm{m}_{r}$, heat that must be removed from the receiver reduces $n$ by more than the same amount of heat being removed from the supply tank. This effect is more pronounced if there is a large temperature difference across the pump. From this table, it is clear that the MP is the most effective pump and that the subcritical FEP is the least effective.

In addition to the losses that result from ideal pumps, there are losses within the pump and within the rest of the transfer system that affect $\eta$. 
The principal losses are listed in Table 2. This table also gives the location where most of the mass loss is expected to occur for each mechanism. Note the internal pump losses that appear in the receiver column. These losses could occur in the supply tank if an appropriate heat exchanger were placed between the pump outlet and the supply tank. The inclusion of the Table 2 losses in (7) could affect the evaluation $6 \mathrm{f}$ the relative merits of the different pumps.

The supercritical FEP has not been experimentally demonstrated and appears to be inconsistent with the usual two-fluid model. To expand on the last point, consider a system of two FEPs in series (Fig. 9). Heat is applied only to the downstream pump. In the region between the pumps, thermal equilibrium between the superfluid and normal components is established. Assuming that the pumps are ideal superleaks, it is simple to show from (1) and (2) that the outlet pressure and heater power are

$$
P_{2}-P_{0}=\int_{T_{0}}^{T_{2}} \rho S d T
$$

and

$$
Q_{2}=\dot{m} S\left(T_{2}\right) T_{2}
$$

respectively. These relations are the same as would be expected if only a single pump were used. Therefore, the fountain (1) and mechanocaloric (2) effects remain valid when "local thermodynamic equilibrium" exists. Also, the turbulence required for this supercritical state would result in frictional 
losses. Such losses are predicted from the two-fluid model ${ }^{15}$ and have been observed, 14,16 but are not included in (6). In any case, the current transfer-system design is based on a pump that will operate at subcritical velocities--not in the turbulent regime. Thus, it is the behavior of the subcritical FEP that is important for the current application.

\section{Multistage fountain-effect pumps}

A potential drawback of the FEP is its inability to generate high pressures (while this does not appear to be a drawback for a space-based transfer system, it could be for other applications). An ideal FEP produces the maximum pressure when the constant chemical potential curve intersects the lambdaline (3). In an actual pump this is reduced by internal losses 14,15 as shown in Fig. 4. An MP has no such limit; rather, it is limited by the design of its impeller. However, it is possible to increase the pressure output of an FEP by staging the pumps (as shown diagramatically in Fig. 10(a)). The nomenclature used in this figure is the same as that used in Ref. 17. A vortex tube and a heat exchanger is located between the two stages. The vortex tube ensures that the flow is locally turbulent, thereby reducing the thermoconductivity between the first stage and the heat exchanger. In analyzing this pump, we will make the assumptions that the pumps are ideal superleaks, that the heat contributes only to the mechanocaloric effect, that there is no pressure drop in the vortex and heat exchanger tubes, and that the heat exchanger is $100 \%$ effective. It is then easy to show from (1) and (2) that the total pressure rise is 


$$
P_{2}-P_{0}=\int_{T_{0}}^{T_{1}} \rho S d T+\int_{T_{0}}^{T_{2}} \rho S d T
$$

and the heat input is

$$
Q_{\text {tot }}=\dot{m}\left[S\left(P_{1}, T_{1}\right) T_{1}+S\left(P_{2}, T_{2}\right) T_{2}\right]
$$

These are the effective fountain and mechanocaloric effects, respectively. The total heat load on the supply tank is

$$
Q_{S}=\dot{m}\left[S\left(P_{0}, T_{0}\right)+S\left(P_{1}, T_{0}\right)\right] T_{0}+\int_{T_{0}}^{T} C_{P} d T
$$

The first two terms on the right side of (12) are derived from the mechanocaloric effect on the upstream side of the pumps and the last term represents the heat extracted from the flow by the heat exchanger. The net effect of staging the pumps is to increase the pressure at the expense of increased power consumption. In principle any number of stages can be used to generate large pressure differences. A pump consisting of $n$ stages would have ( $n-1)$ heat exchangers between the stages. If the output temperature of each stage is $\mathrm{T}_{1}$, and the components are ideal, then the pressure dependencies of $\mathrm{S}$ and $\mathrm{C}_{\mathrm{P}}$ can be neglected, producing for an $\mathrm{n}$-stage system

$$
P_{n}-P_{0}=n \int_{T_{0}}^{T_{1}} \rho S d T
$$




$$
\begin{aligned}
& Q_{\text {tot }}=\dot{m} n S\left(T_{1}\right) T_{1} \\
& Q_{S}=\dot{m}\left[n S\left(T_{0}\right) T_{0}+(n-1) \int_{T_{0}}^{T_{1}} C_{P} d T\right]
\end{aligned}
$$

The preceding equations (10-15) ignore the effects of losses within the pump. A multistage FEP would be expected to have the same loss mechanisms that a single-stage FEP has. ${ }^{15}$ These losses reduce the pressure head $\left(P_{n}-P_{0}\right)$ and increase the heat required $\left(Q_{\text {tot }}\right)$ to produce a given flow. In addition, the multistage FEP performance is affected by the effectivenesses of the heat exchangers. Because of the heat-exchanger ineffectiveness, the temperature will increase at the inlet of the second and subsequent stages. There are four effects at this increase: 1) For the same pressure head for each stage (except the first), the outlet temperature will be higher; i.e., (13) must be rewritten as:

$$
P_{n}-P_{0}=\int_{T_{0}}^{T_{1}} \rho S d T+(n-1) \int_{T_{0}^{\prime}}^{T_{1}} \rho S d T
$$

where $T_{0}^{\prime}$ is heat-exchanger-outlet temperature and $T_{0}^{\prime}>T_{0}$. Thus, for a given total-pressure head, $T_{1}$ must be increased. 2) The maximum possible pressure (as defined by (3) will be reduced because increasing the inlet temperature to $T_{0}^{\prime}$ decreases $\mu$, which causes $P$ to decrease. (This is the 
result of the thermodynamic relation: $d \mu=-S d T+V d P$.$) 3) Q_{\text {tot }}$ is also increased because of the increase in $T_{1}$. 4) $Q_{S}$ will increase and become

$$
Q_{S}=\dot{m}\left\{S\left(T_{0}\right) T_{0}+(n-1)\left[S\left(T_{0}^{\prime}\right) T_{0}^{\prime}+\int_{T_{0}^{\prime}}^{T_{1}} C_{P} d T\right]\right\}
$$

(Raising $T_{0}^{\prime}$ increases $Q_{S}$ because the mechanocaloric term increases more than the $\mathrm{C}_{\mathrm{P}}$ term decreases.)

\section{Conclusion}

Two different types of pumps are being developed for resupplying liquid helium to scientific instruments in orbit. These pumps are the MP and the FEP. The operating characteristics of the two pumps are quite different; but both appear to meet the operating requirements. The FEP has the advantage of simplicity and therefore should be reliable, although the MP has also proven reliable in testing to date. Testing and analysis have shown the MP to be more efficient; however, a proposed supercritical operating mode of the FEP could make it the more efficient pump. Such a pump has not yet been demoristrated.

The effect of pump efficiency on the transfer effectiveness of the entire system is not expected to be large. During development testing, a useful comparative tool is the cyclic transfer effectiveness. This is the ratio of the mass that is transferred and conditioned to the initial mass when the transfer is treated as a thermodynamic cycle (starting and finishing at the same temperature and pressure). When this ratio is applied to idealized pumps, the MP is the most effective, and the simpler, subcritical FEP is the 
least effective. The relative performance of these pumps will change if losses are included. The cyclic transfer effectiveness is a useful tool but it may not reflect the effectiveness of a real system. In a real transfer system, the temperatures in the supply tank and in the receiver will, in general, not be the same and may vary with time. The effects of this variance have not been considered.

The MP has the advantage of being able to generate higher pressures (with a suitably designed impeller). For an FEP to generate higher pressures, a multistage FEP could be used. Such a pump would allow greater pressures at the expense of reduced efficiency.

This paper has reviewed the state of development of pumps for the orbital resupply of liquid helium. The final choice between the different pumps cannot be made until their respective transfer effectivenesses are measured in space. 


\section{References}

1 Kittel, P. AIAA 20th Thermophysics Conference (Williamsburg, VA, 1985) A.TAA-85-0959

2 Dipirro, M. J., and Castles, S. H., Cryogenics 26 (1986) 84

3 Nast, T. C., Frank, D., Liu, C. K., and Parmley, R. T., Cryogenics 26 (1986) 78

4 Mord, A. J., Urbach, A. R., Poyer, M. E., Andreozzi, L. C., Snyder, H. A., Hermanson, L. A., Blalock, W. R., and Kelly, T. K. Cryogenics 26 (1986) 68

5 MoConnell, P. M. Liquid helium pumps, NBSIR 73-316 (1973)

6 Ludtke, P. R. Performance characteristics of a liquid helium pump, NBSIR $75-816$ (1975)

7 Arp, V. A comparison of centrifugal and fountain effect pumps, cryogenics 26 (1986) 103

8 Steward, W. G. A centrifugal pump for liquid helium, Cryogenics 26 (1986) 97

9 Frederking, T. H. K., Yuan, S. W. K., and Carandang, R. M. Fountain effect pump phenomena for liquid helium transfer: Thermodynamic system studies, Cryogenics 26 (1986) 93

10 Brooks, W. F., Kittel, P., Lee, J. H., and Ng, Y. S. Proc. Satellite services workshop II, Report JSC-20677, 1985

11 Brooks, W. F. Flight demonstration of helium transfer in space, this conference, April 1986

12 Kittel, P. Cryogenics 26 (1986) 59 
13 Wilks, J. The Properties of Liquid and Solid Helium, McGraw-Hill, New York, 1965

14 Keller, W. E. Helium-3 and Helium-4, Plenum Press, 1969

15 Kittel, P. 11th Int. Cryo. Engin. Conf. (Berlin, 1986)

16 Kasthurirengan, S., Schotte, U., Denner, H. D., Szucs, Z., and Klipping, G. Cryogenics 25 (1985) 518

17 Srinivasan, R., and Hofmann, A. Cryogenics 25 (1985) 642

18 Van Sciver, S. W. Adv. Cryo. Engin. 29 (1984) 315 
Table 1 Pump comparison

\begin{tabular}{|c|c|c|c|c|}
\hline & Path on & Heat & \multicolumn{2}{|c|}{ Mass $\operatorname{loss}^{b}$} \\
\hline Pump & S-T diagram ${ }^{\mathrm{a}}$ & influx ${ }^{b}$ & $\begin{array}{c}\text { In receiver } \\
\left(m_{r}\right)\end{array}$ & $\begin{array}{c}\text { In supply } \\
\left(\mathrm{m}_{\mathrm{s}}\right)\end{array}$ \\
\hline
\end{tabular}

supercritical FEP A-B-C-A $\quad \int_{A}^{B} C_{\mu} d T 1-\exp \int_{C}^{A} C_{s a t} / L d T \quad 0$

MP $\quad A-B^{\prime}-C^{\prime}-A \quad 0 \quad 1-\exp \int_{C^{\prime}}^{A} C_{s a t} / L d T \quad 0$

subcritical FEP A-A'-A"-B-C-A $S\left(T_{B}\right) T_{B} \quad 1-\exp \int_{C}^{A} C_{s a t} / L d T \quad S\left(T_{A}\right) T_{A} / L$

a - Fig. 8

b - per unit mass pumped $\left(m_{P}\right)$, the limits of integration and subscripts refer to the labeled points in Fig. 8 


\begin{tabular}{|c|c|c|c|}
\hline \multirow[t]{2}{*}{ Pump } & \multirow[t]{2}{*}{ Loss mechanism ${ }^{a}$} & \multicolumn{2}{|c|}{ Location of principal mass loss } \\
\hline & & Supply & Receiver \\
\hline \multirow[t]{2}{*}{$\mathrm{MP}^{\mathrm{b}}$} & motor & $x$ & \\
\hline & pumping & & $x^{c}$ \\
\hline \multirow[t]{3}{*}{ FEP $^{d}$} & thermal conductivity & $\mathrm{x}$ & \\
\hline & heater location & & $x^{c}$ \\
\hline & mutual friction & & $x^{c}$ \\
\hline \multirow[t]{2}{*}{ both ${ }^{e}$} & transfer line & & $x$ \\
\hline & Dewar parasitics & $\mathrm{x}$ & $x$ \\
\hline
\end{tabular}

\footnotetext{
a - these losses are not necessarily proportional to mass flow b - refs. $5,6,7,8,9$

c - assuming no heat exchange between supply tank and pump outlet

d - refs. 14,15

e - refs. $2,3,4,10,11$

f - may increase during transfer
} 
Figures

Fig. 1 Cross-sectional view of the mechanical pump being tested. Adapted from Ref. (6).

Fig. 2 Predicted performance characteristics of the mechanical pump. Adapted from Ref. (8).

Fig. 3 Representation of an FEP. A heat source on the downstream side of the porous plug drives the flow.

Fig. 4 Representation of the T-P operating characteristics of an FEP. Adapted from Ref. (14).

Fig. 5 Efficiency of an MP (_-) and an FEP (-----). From Ref. (1).

Fig. 6 Conversion loss in the receiver as a result of evaporative cooling. The curves represent the volume percent lost in cooling from various temperatures (labeled on curves to a final tgemperature). From Ref. (1).

Fig. 7 Comparison of transfer effectiveness for an MP (_ (....-) for the system described in Ref. (2).

Fig. 8 Entropy-temperature diagram showing the idealized cycles for the

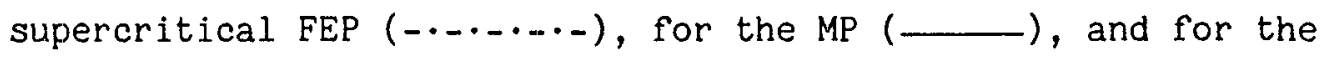
subcritical FEP (-----). All of the cycles start at A at saturated conditions. During the cycles the liquid is taken from the supply tank and pressurized ( $B$ or $\left.B^{\prime}\right)$. It flows into the receiver ( $C$ or $\left.C^{\prime}\right)$ and is recooled to $A$.

Fig. 9 Representation of two FEPs operating in series. The pump is activated by a single heater on the downstream side of the second stage.

Fig. 10 (a) Representation of a two-stage FEP with an intermediate heat exchanger. Each stage has its own heater. (b) Also shown is the temperature/pressure operating characteristics of such a pump. 


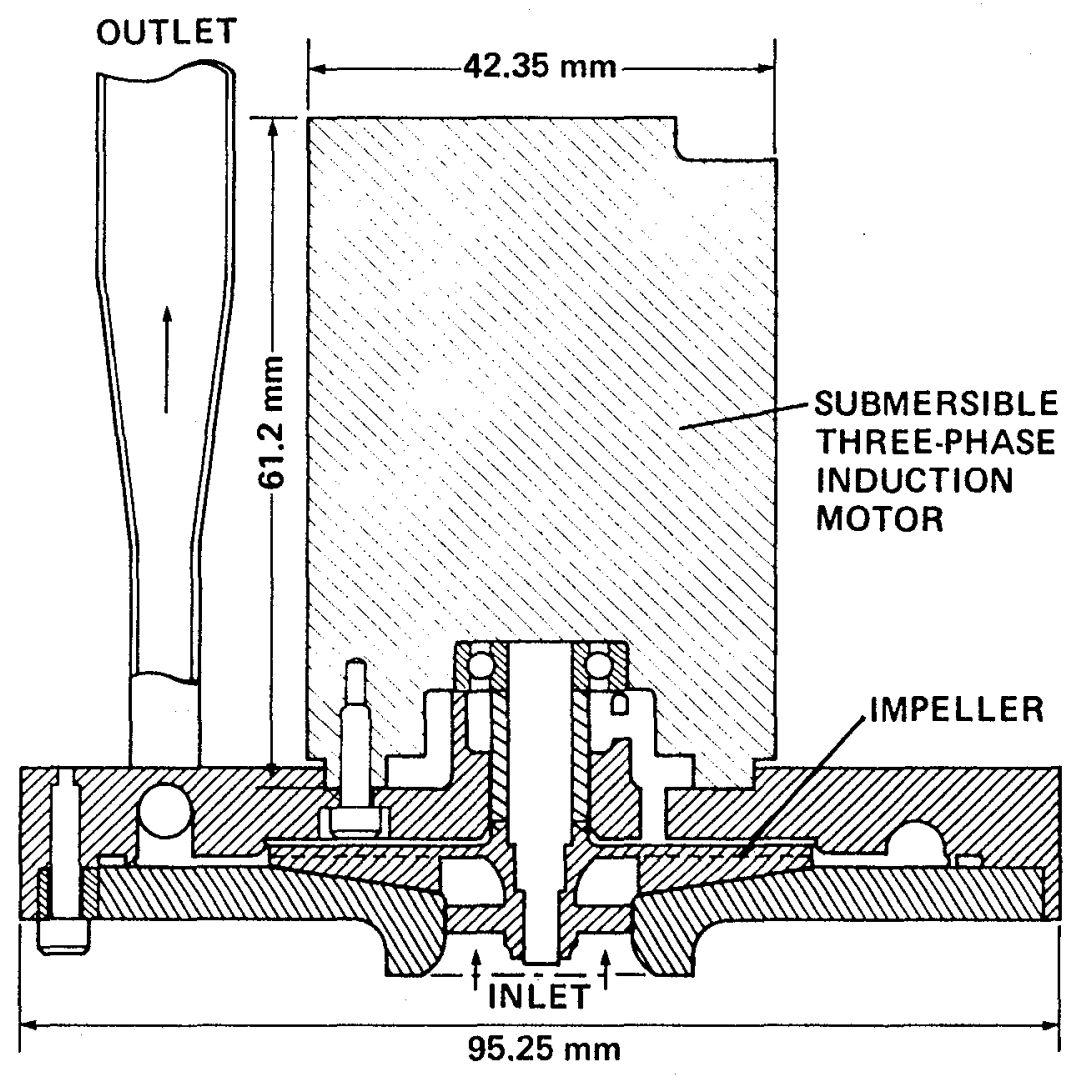

Fig. 1 


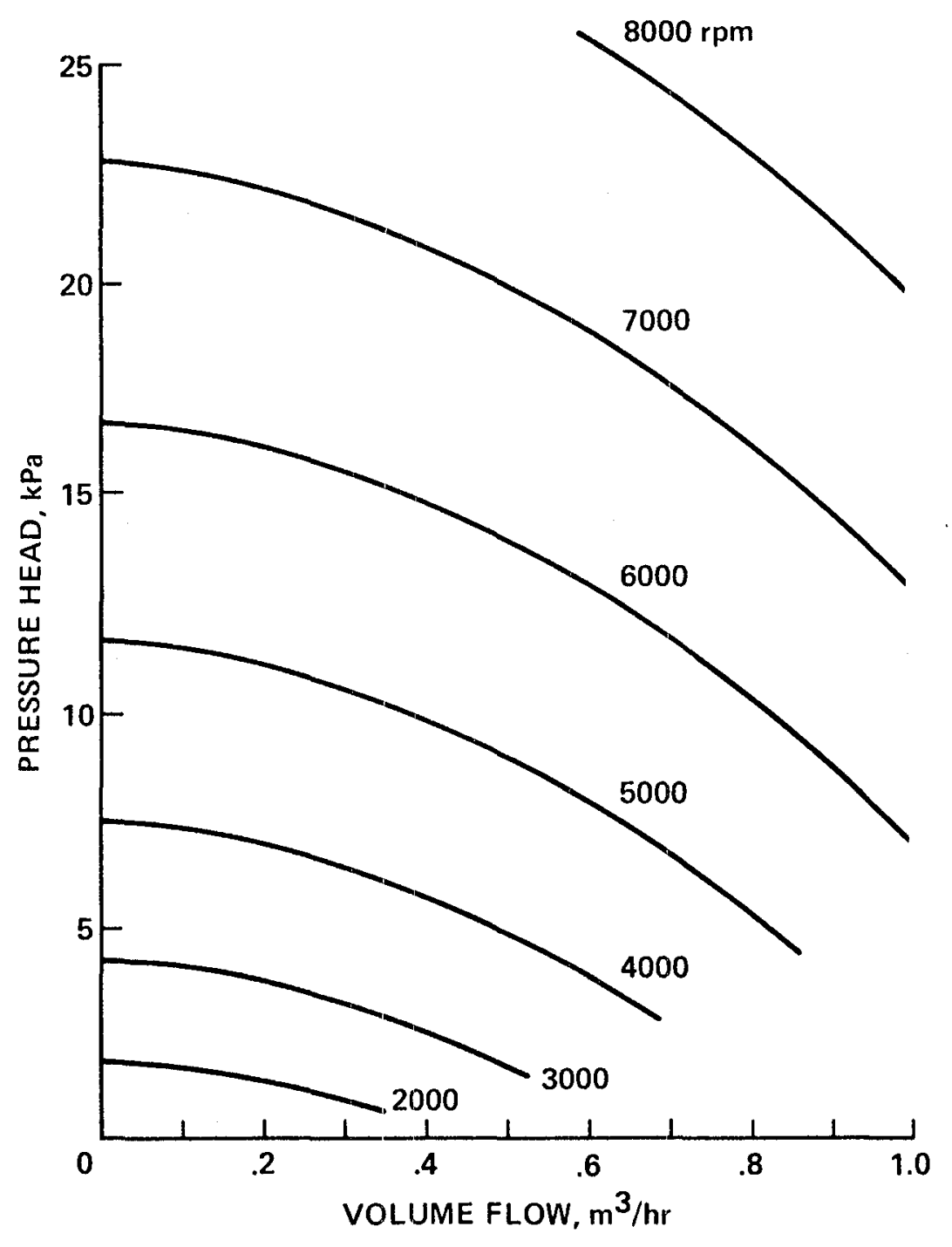

Fig. 2 


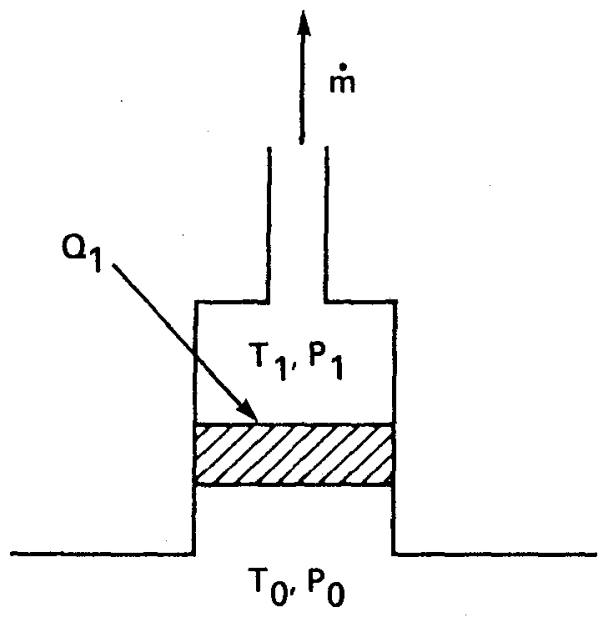

Fig. 3 


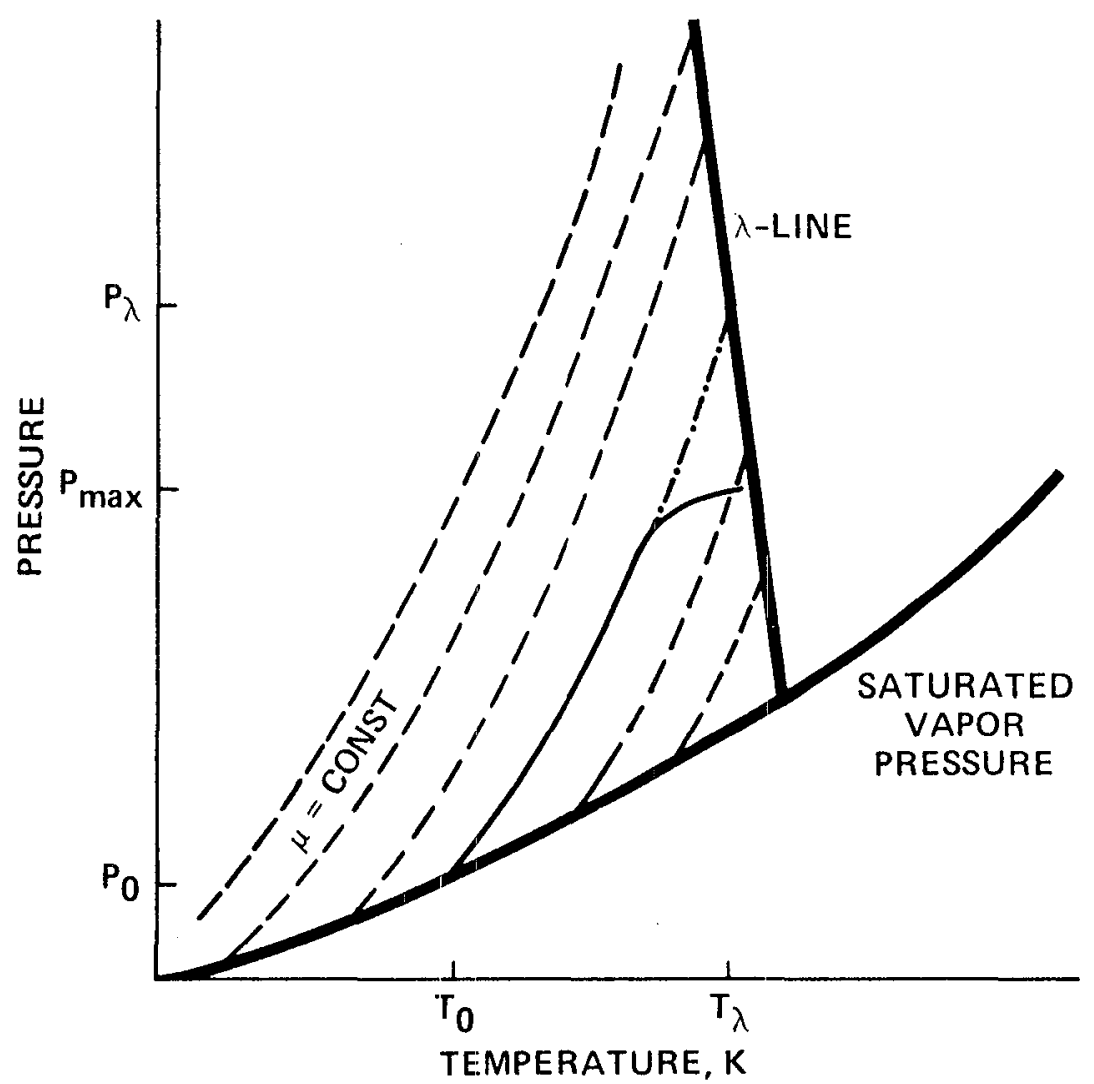

Fig. 4 


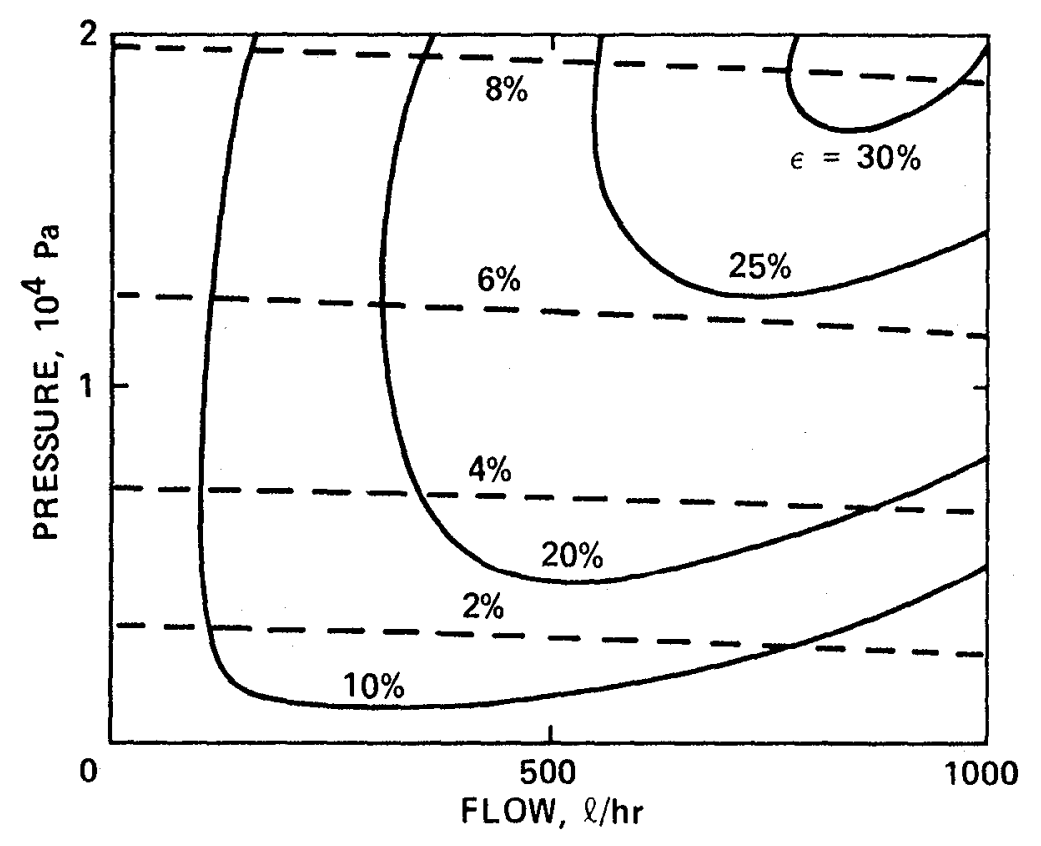

Fig. 5 


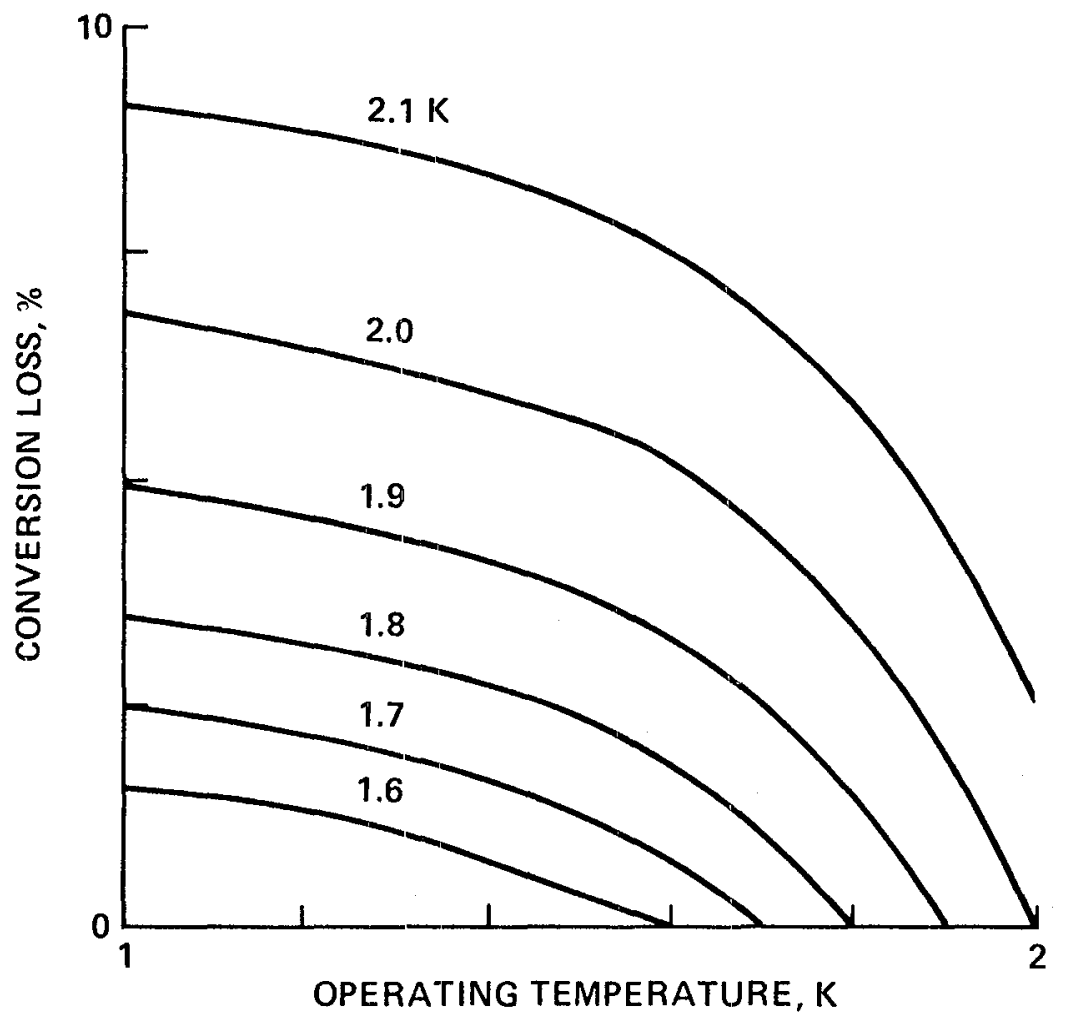

Fig. 6 


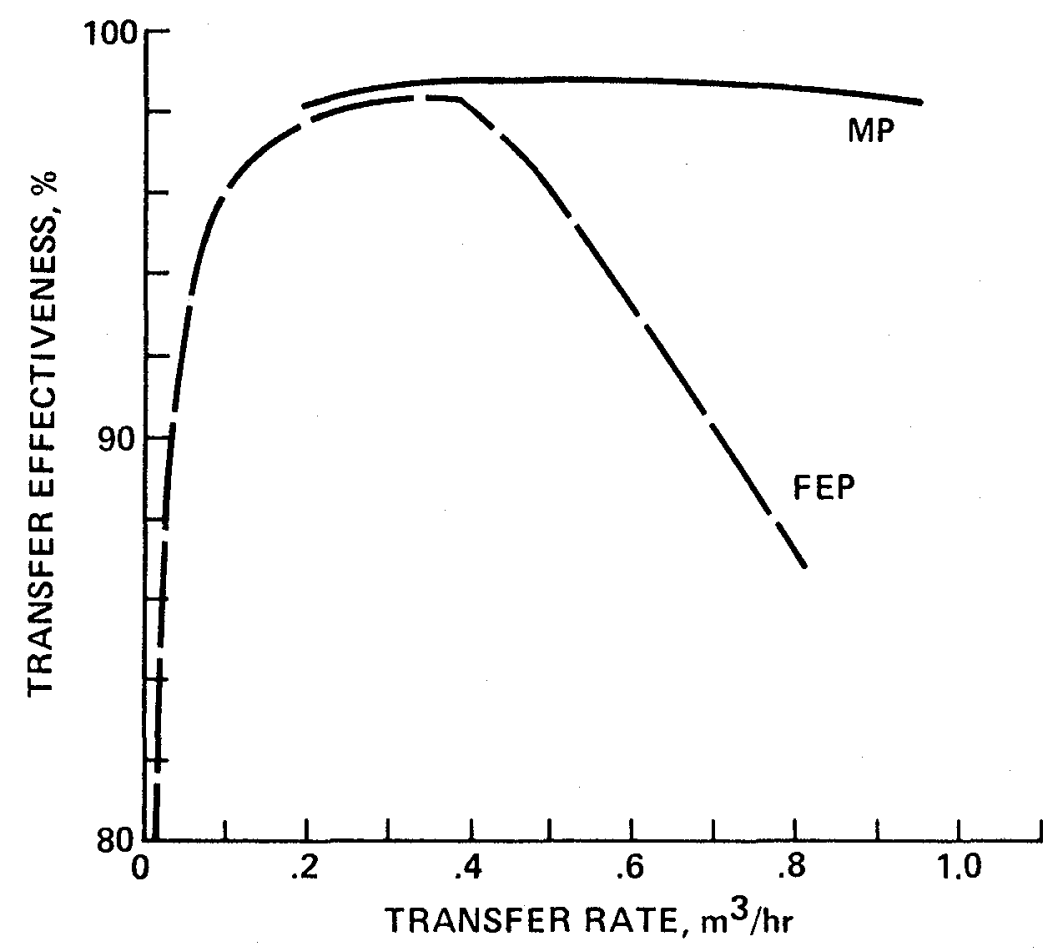

Fig. 7 


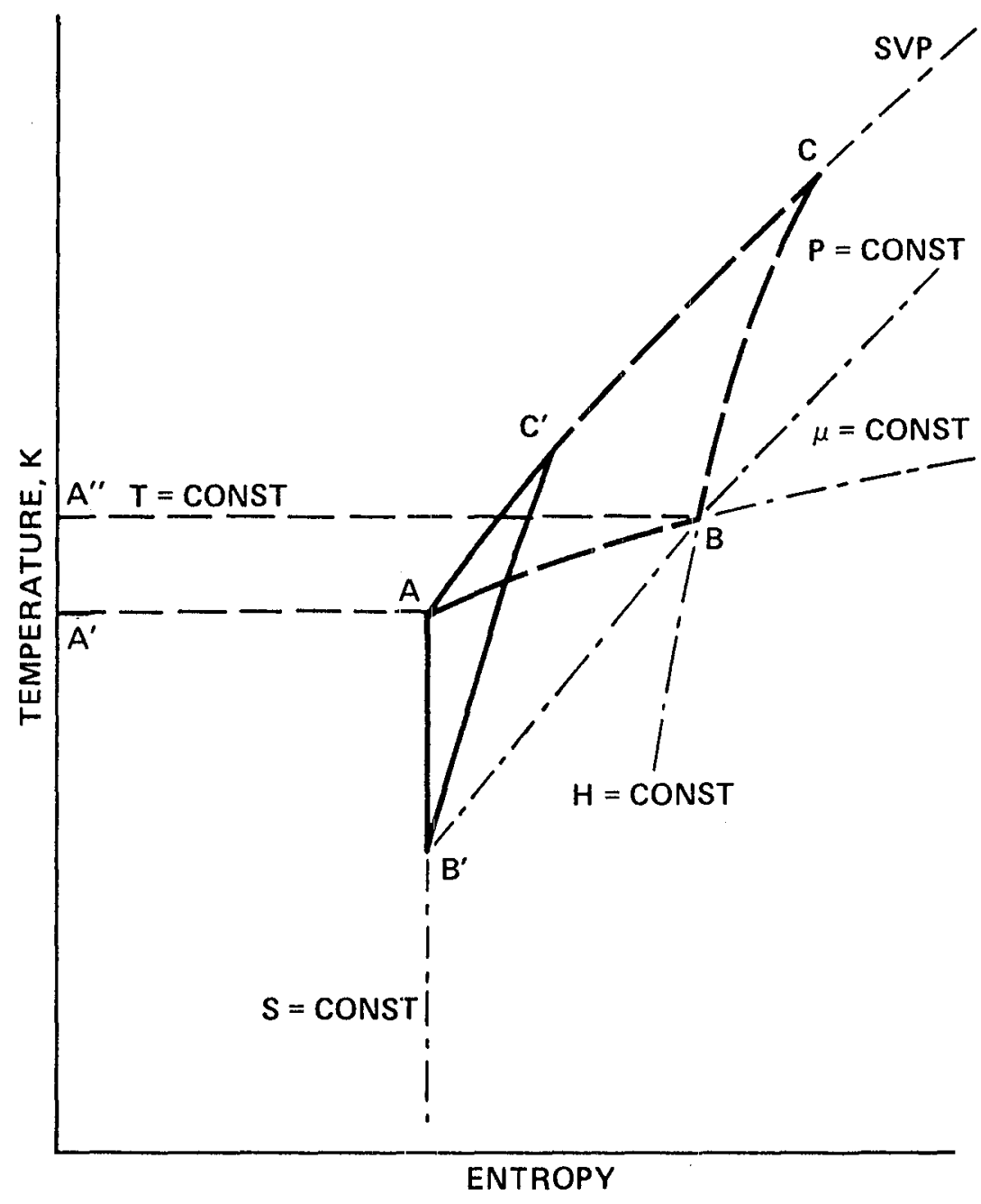

Fig. 8 


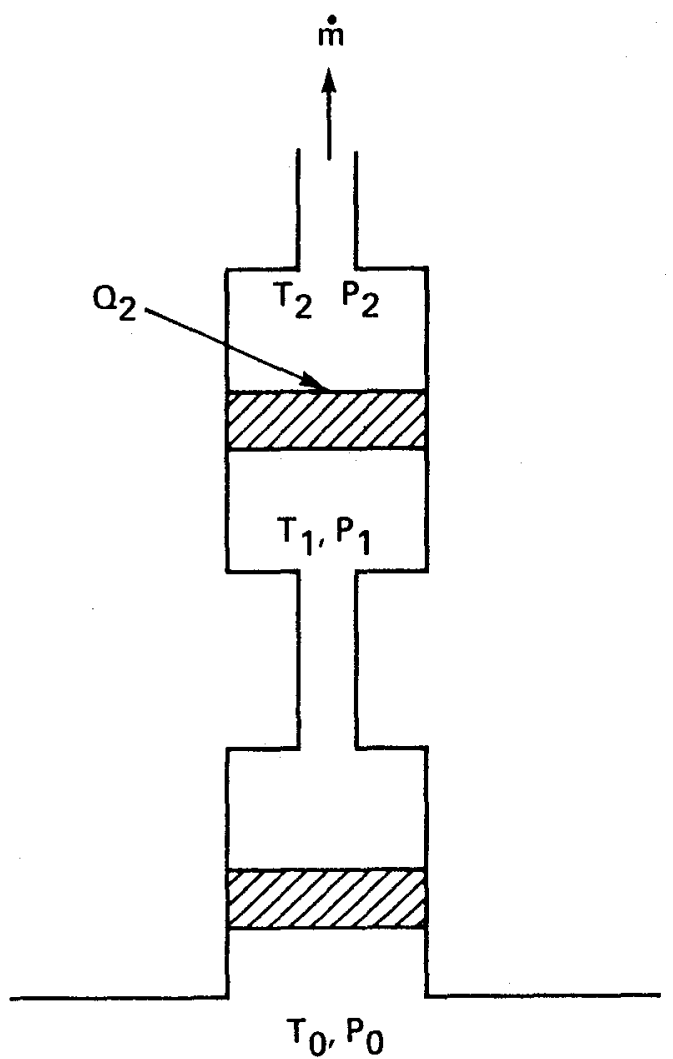

Fig. 9 


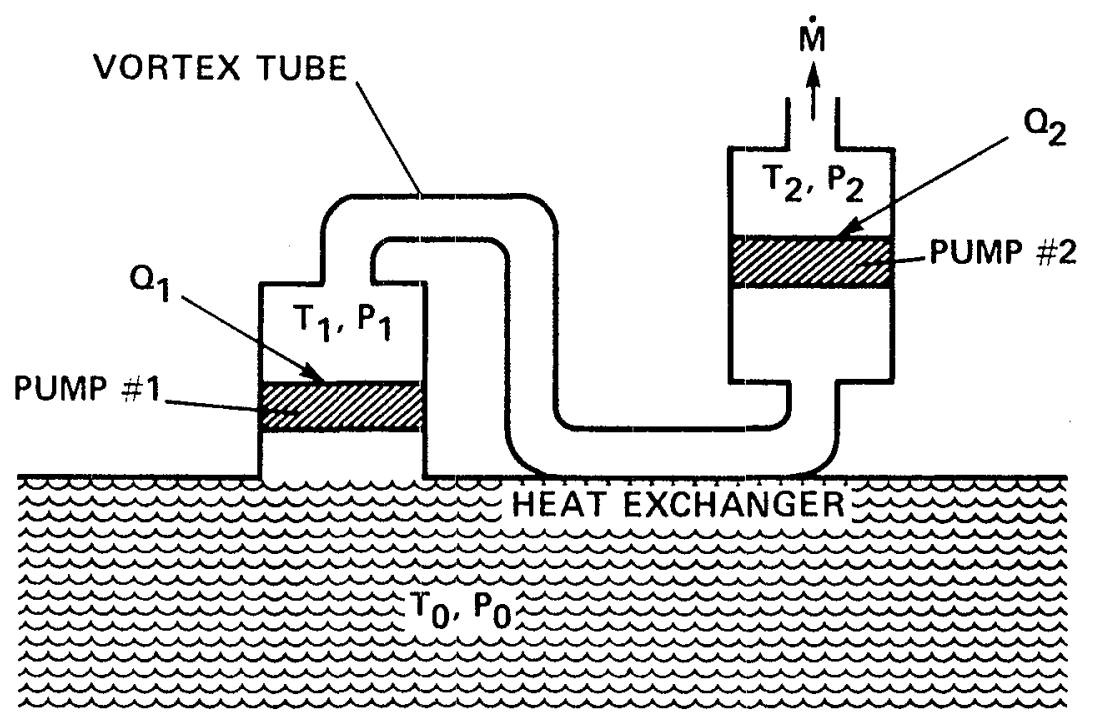

Fig. 10 Top 


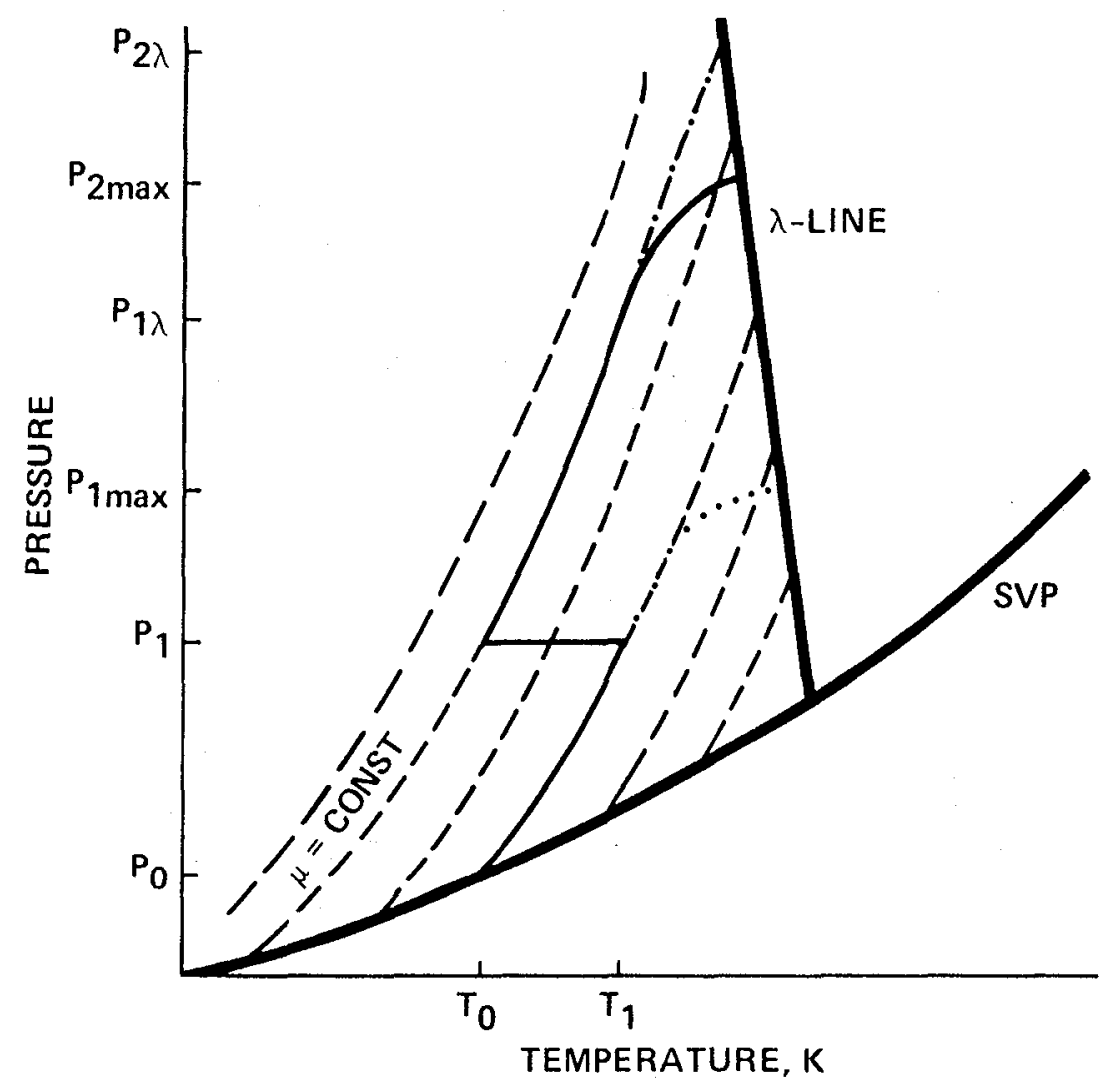

Fig. 10 Botiom 


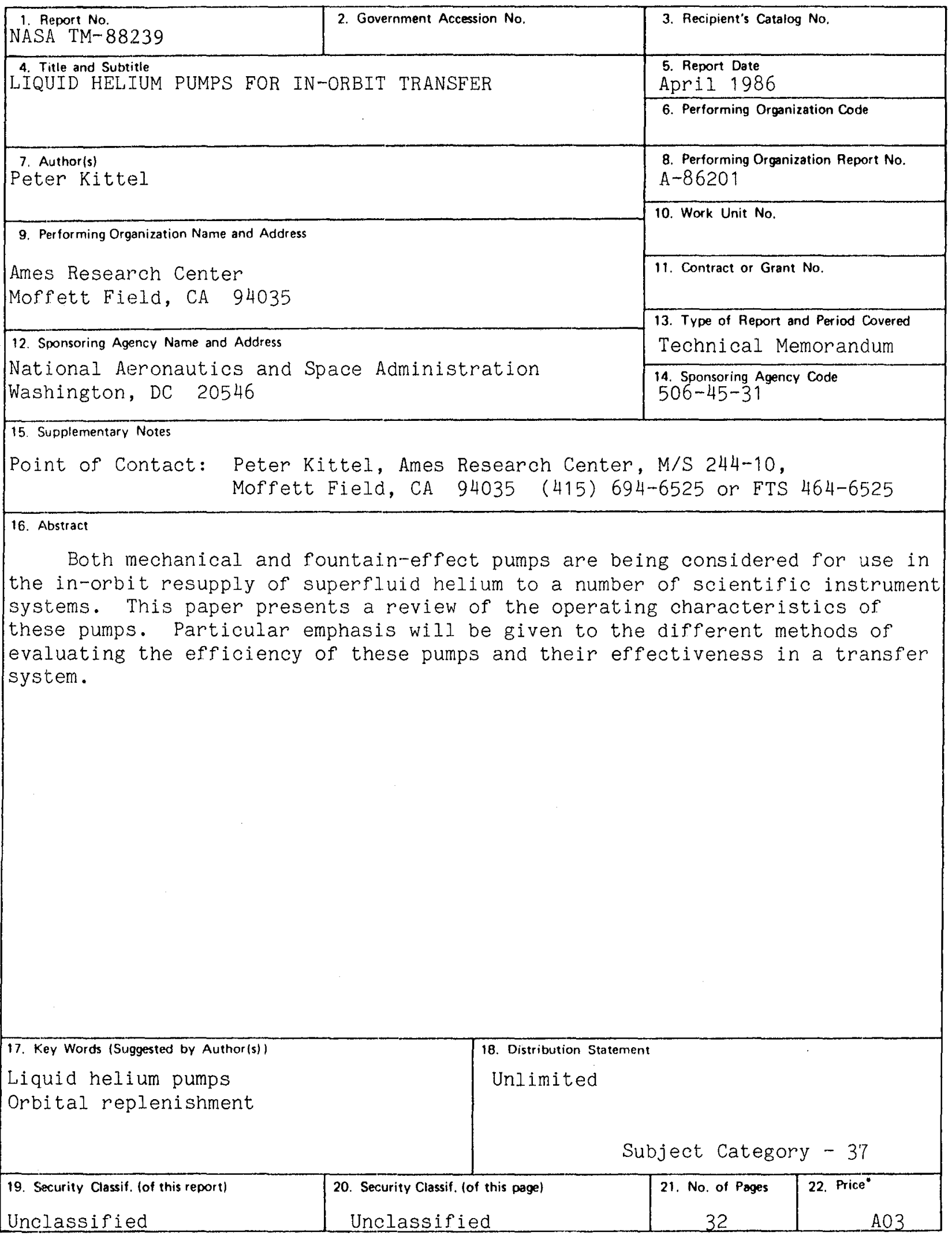

"For sale by the National Technical Information Service, Springfield, Virginia 22161 
End of Document 